\title{
Emerging technologies in ambient systems
}

\author{
Ansar-Ul-Haque Yasar $^{1}$ - Haroon Malik ${ }^{2}$ Elhadi M. Shakshuki ${ }^{3}$. \\ Zahoor Khan ${ }^{4}$
}

Published online: 14 May 2016

(c) Springer-Verlag Berlin Heidelberg 2016

This special issue is based on selected papers from both the 10th International Conference on Future Networks and Communications, and the 12th International Conference on Mobile Systems and Pervasive Computing (MobiSPC) that were held on August 17-20, 2015, Belfort, France. The conference attracted a good number of scientific papers that contributed in the areas of pervasive and ambient information systems. All the papers, selected for this special issue, have been extended from their original versions and underwent two rounds of rigorous peer-review process. Based on the reviewers' feedback, as well as the evaluations of the Guest Editors. The accepted papers cover interesting works on new developments in ambient and networked systems such as internet-of-things (IoT), wireless sensors network (WSN), Grid and Cloud systems.

The first paper by Wagoner et al. is entitled "A task manager using an ontological framework for a HARMSbased system". The authors presented initial steps in the development of a theoretical task manager that uses an ontological framework for a human, agent, robot, machine, sensor for a HARMS-based multi-agent system. The task manager is an extension of a robust dialogue manager that enables natural interaction between humans and the multiagent system. The task manager retrieves information

Elhadi M. Shakshuki

elhadi.shakshuki@acadiau.ca

1 Transportation Research Institute, Hasselt University, Hasselt, Belgium

2 Weisberg Division of Computer Science, Marshall University, Huntington, WV, USA

3 Jodrey School of Computer Science, Acadia University, Wolfville, Canada

4 Higher Colleges of Technology, Fujairah, UAE about a desired task the human wants completed from the dialogue manager. The task manager uses an ontology to break the task into subtasks and finds a capable agent to complete each subtask. An experiment was conducted that combined the dialogue manager and task manager. The results showed that the initial developments have a success rate of $83.3 \%$. While the user is unlimited in the grammatical structure of the input, the experiment did show, however, that the user is limited by the vocabulary that can be used because of the strict limitations of the ontology.

The second paper by Xue et al. is entitled "Towards horizontally scalable apps". The authors introduced ways to enable apps to horizontally scale across multiple mobile devices by accessing all available hardware and software resources. The authors presented an evaluation of the micro-services pattern in apps for mobile devices that use the IoT communication protocol CoAP in different WIFI environments and different encryption methods. They examined the I/O performance of CoAP in two different wireless settings and presented the data. The evaluation with two average mobile devices (smartphone and tablet) showed that the maximum throughput is around $800 \mathrm{~KB} / \mathrm{s}$ at the maximum payload size of 1024 bytes and at least 330 subscriptions per second can be achieved. Additionally, the authors observed that the 128 bytes payload size seems ideal in terms of subscriber response time and throughput. Applied AES encryption to CoAP communications, the IO throughput goes down not more than $170 \mathrm{~KB} / \mathrm{s}$ and there is no big performance difference between different key size encryption. While CoAP uses UDP as a transport protocol, it is possible to use non-IP based transport protocols.

The third paper by Boulekrouche et al. is entitled "Toward integrating grid and cloud-based concepts for an enhanced deployment of spatial data warehouses in cyberphysical system applications". The authors proposed a 
multi-agent based approach to distribute the spatial extracttransform load (SETL) processes over a grid of computing resource and improve the management of spatial data warehouse (SDW) with cloud computing tools. The approach made use of cloud computing techniques to shorten the spatial data processing time and reduced resource consumption. The approach is also intended to enabling a joint use of real-time and archive data within the context of SDWs for cyber physical system (CPS) applications for personalized reporting and visualization of services envisioned to the decision-makers who are using the same CPS application.

The fourth paper by Sheltami et al. is entitled "Continuous object detection and tracking in wireless sensor networks". The authors proposed an energy efficient algorithm for continuous object tracking and detection. The algorithm is an improved hybrid algorithm integrating the detection techniques of two algorithms, i.e., continuous boundary monitoring (COBOM) and detection and monitoring for continuous objects (DEMOCO). Monitoring continuous objects pose new challenges due to their nature and characteristics of changing in size and shape, shrinking and expanding, splitting into multiple objects, or merging of multiple objects into one object. Continuous objects tracking and detection require extensive communication, which consumes a considerable amount of network energy. Towards this end, authors also introduce a new data structure for reporting data. This new data structure reduces the communication cost of the overall algorithm without compromising the accuracy for reconstructing the boundary of a continuous object at the base station. A concept for differentiating between the holes in the phenomenon and overall phenomenon changes, at the base station level, is also introduced which provides additional information to the user as an added improvement while maintaining the high accuracy and efficiency.

The fifth paper by Malik et al. is entitled "Connecting the dots: anomaly and discontinuity detection in large-scale systems". The authors propose a technique that helps analyst in datacenters and cloud systems automatically identify discontinuities in the performance data. Discontinuity is a change in a time-series pattern that persists (but does not reoccur) since the measurement taken before the discontinuity may be irrelevant. Detecting discontinuities in performance data of a data center improves forecasts. The authors showed how simple statistical techniques could be used to identify discontinuities in large performance data using an industrial system as well as an open source system.

The sixth paper by Nordhal et al. is entitled "A lightweight data interchange format for Internet of things with applications in the PalCom middleware framework". Authors presented a new data interchange format PalCom Object Notation (PON), which supports combinations of text and binary information to be included in the same message. In comparison to JSON, PON supports some additional value types, being closely coupled with the types of the programming language Java, and in particular supports direct handling of binary information. It is designed for IoT situations where communication is between devices, but is still textual and thus human readable, apart from when used with binary payloads.

The guest editors would like to take this opportunity to thank all the authors for the efforts they put in the preparation of their manuscripts and for their valuable contributions. We wish to express our deepest gratitude to the referees who provided very useful and thoughtful feedback to our authors. Our sincere thanks go to the Editor-in-Chief for his kind help and support. 